\title{
Radiological man- agement of skeletal metastases, multiple myeloma and lymphoma
}

\section{Maximilian F Reiser MD}

In malignant tumors distant metastases are of utmost importance for the choice among different treatment options. Depending upon the primary tumor, lung, liver and the skeletal system are frequent sites of distant metastases. The diagnosis of multiple myeloma is based on laboratory findings. For staging of this disease, plain radiograms of the skeletal system are obtained in order to assess skeletal involvement and fracture risk, especially of the spinal column. Lymphoma usually originates from the lymph nodes and staging is focussed on the visualization of the extent of lymph node involvement. In advanced stages of malignant lymphoma, the skeletal system may also be involved.

Skeletal metastases, multiple myeloma and lymphoma are mainly distributed via the arterial circulation and seed into the bone marrow. As the neoplastic cells proliferate the normal bone marrow elements are replaced. Due to its better vascularization, the red marrow is more frequently affected. In advanced stages of bone marrow involvement, the yellow marrow is replaced by red marrow due to the demand in production of blood cells (reconversion) and peripheral skeletal elements are also prone to seeding of metastatic cells.

Various imaging modalities are used for the diagnostic workup of skeletal manifestations of metastases, multiple myeloma and lymphoma. Their relative value depends on the principle of the particular imaging modality on one hand, and the biological properties of the underlying malignancy on the other hand.

Plain radiography, CT, MRI, bone scanning and PET are adequate methods for the diagnosis of these disorders and frequently a combination of these techniques is employed. Recently whole body MRI, whole body CT and CT-PET have been described.

\section{Radiography}

In order to detect bone destructions on plain radiograms about $30 \%$ of the bone substance has to be removed. Cortical destructions are more apparent than purely trabecular lesions. In preexisting osteoporosis, lysis of the bone is more difficult to assess. Numerous reports have shown that radiography is less sensitive than bone scanning, PET, CT and MRI in the detection of skeletal metastases, multiple myeloma and lymphoma. When neoplastic cells are invading the bone marrow without or with minor destruction of the bone trabecular, no radiographic abnormalities can be detected.

Radiography, on the other hand, allows one to differentiate lytic, sclerotic and mixed pattern of bone metastases and to establish differential diagnoses when other imaging modalities, e.g. bone scanning show suspicious findings.

\section{Computed tomography}

With single slice CT only limited anatomical areas could be examined and CT was used to clarify ambiguous results obtained with other modalities. Multislice CT enables one to scan large portions of the body within short acquisition times and with thin collimation. Multiplanar reconstructions allow one to display the skeletal system in imaging planes adequate for diagnostic reading, e.g. Sagittal and coronal MPR's of the spine and coronal MPR's of the pelvis. CT is also superior to radiography in the assessment of bones at risk of fracture. In the spine endplate fractures not visualized on radiograms can be detected. Recently it was published that MSCT with coronal and sagittal MPR's is efficient in staging patients with multiple myeloma by demonstrating nodular destructions and also tiny trabecular and cortical defects. Replacement of bone marrow by neoplastic tissue results in an increase of density as demonstrated by CT. This is better recognizable within the fatty marrow than the red marrow and does not allow for a reliable diagnosis. 


\section{Magnetic resonance imaging (MRI)}

MRI has a unique soft tissue contrast and enables for the election of diffuse and focal bone marrow infiltration. T1-weighted spin echo, STIR and fat sat proton density and T2weighted pulse sequences are most sensitive for the detection of neoplastic infiltration of the bone marrow. Numerous reports underline the superior sensitivity of MRI in the detection of skeletal metastases, and manifestations of multiple myeloma and lymphoma respectively. Whole body MRI has been performed in comparison to bone scanning in the detection of skeletal metastases. The total number of metastases detected with MRI was higher than that of bone scanning. MRI was particularly useful in the spine and pelvis, whereas bone scanning performed better in the skull and ribs. In addition to skeletal metastases MRI was able to detect metastases to other organs with high accuracy.

In multiple myeloma MRI enables one to differentiate diffuse, nodular and mixed nodular and diffuse skeletal manifestations. For the assessment of diffuse bone marrow infiltration by myeloma, contrast enhanced MRI proved to be very valuable. Enhancement within the bone marrow of $20 \%$ and more is indicative of moderate to high grade myeloma infiltration. Low grade infiltration can not be diagnosed with MRI and bone biopsy is required. In addition to clinical parameters MRI findings are an independent prognostic factor in patients with multiple myeloma.

\section{Bone scanning and PET}

In bone metastases with sclerotic or mixed pattern, bone scanning is very sensitive, whereas purely lytic metastases (renal cell carcinoma, thyroid cancer, multiple myeloma) are frequently not associated with tracer accumulation. While bone is highly sensitive, its specificity is limited. Therefore, unless findings are specific for neoplastic infiltration, correlation with other imaging techniques is required. FDG-PET is correlated to increased metabolism of tumor cells and enables one to diagnose increased cell turn over of neoplasms. However, false-positives and negatives have to be ruled out.

\section{Suggested reading}

1. Bollow M, Knauf W, Korfel A, Taupitz M, Schilling A, Wolf KJ, Hamm B. Initial experience with dynamic MR imaging in evaluation of normal bone marrow versus malignant bone marrow infiltrations in humans. J Magn Reson Imaging 1997 Jan-Feb; 7(1): 241-250.

2. Byun WM, Shin SO, Chang Y, Lee SJ, Finsterbusch J, Frahm J. Diffusion-weighted MR imaging of metastatic disease of the spine: assessment of response to therapy. AJNR Am J Neuroradiol 2002 Jun-Jul; 23(6):906-912.

3. Linden A, Zankovich R, Theissen P, Diehl V, Schicha H. Malignant lymphoma: bone marrow imaging versus biopsy. Radiology 1989 Nov; 173(2): 335-339.

4. Marcuzzi DW, Cohen EK. Role of MRI bone marrow imaging in recurrent lymphoma. Can Assoc Radiol J 1989 Aug; 40(4): 216-218 Comment in: Can Assoc Radiol J. 1990 Feb; 41(1): 51 .

5. Nyman R, Rehn S, Glimelius B, Hagberg H, Hemmingsson A, Jung B, Simonsson B, Sundstrom C. Magnetic resonance imaging in diffuse malignant bone marrow diseases. Acta Radiol 1987 Mar-Apr; 28(2): 199-205.

6. Rybak LD, Rosenthal DI. Radiological imaging for the diagnosis of bone metastases. Q J Nucl Med 2001 Mar; 45(1): 53-64.

7. Thompson JA, Shields AF, Porter BA, Olson DO, Rubin E, Kidd P, Fefer A. Magnetic resonance imaging of bone marrow in hairy cell leukemia: correlation with clinical response to alpha-interferon. Leukemia 1987 Apr; 1(4):315316. 